\title{
Influência de fatores contingenciais no desempenho socioeconômico de governos locais
}

\section{Clóvis Fiirst 1}

\section{Ilse Maria Beuren 2}

1 Universidade Estadual do Oeste do Paraná / Departamento de Ciências Contábeis, Cascavel / PR - Brasil

2 Universidade Federal de Santa Catarina / Programa de Pós-graduação em Contabilidade, Florianópolis / SC - Brasil

\begin{abstract}
O desafio desta pesquisa é responder quais elementos caracterizam os fatores contingenciais no setor público e como eles influenciam o desempenho socioeconômico municipal. As principais hipóteses testadas neste estudo analisam se os fatores contingenciais ambiente (contexto externo), tecnologia (processos), estrutura (investimentos) e porte (tamanho) têm influência sobre o desempenho socioeconômico dos governos locais. Para tanto, uma pesquisa documental foi realizada junto aos 399 municípios paranaenses, totalizando 1.995 observações relativas ao período de 2013 a 2017, o que corresponde a um ciclo orçamentário completo. Para a análise dos dados, aplicou-se uma regressão linear múltipla com dados em painel. Os resultados revelam que os fatores contingenciais "ambiente" e "porte" afetam positivamente os resultados socioeconômicos, enquanto "tecnologia” apresenta influência negativa e "estrutura" não apresentou resultados significantes. Com base nesses resultados, conclui-se que os fatores externos, aqueles não controláveis pelos gestores, são os que melhor explicam o comportamento do desempenho socioeconômico dos governos locais investigados. Assim, cabe aos gestores implantar políticas públicas capazes de interferir em seu ambiente e incentivar um porte que contribua para o desempenho socioeconômico e atenda aos objetivos da população. De igual modo, os resultados apresentam implicações teóricas, pois contribuem para a literatura no sentido de acrescentar ao fluxo da pesquisa, fundamentada na Teoria da Contingência, elementos que caracterizam contingências no setor público, no âmbito municipal, como observatório social, grau de dependência, planejamento orçamentário, capacidades do órgão público em manter suas despesas correntes, estrutura de pessoal e de investimentos e porte considerando a receita do município.
\end{abstract}

Palavras-chave: fatores contingenciais; desempenho socioeconômico; governos locais.

\section{Influencia de factores de contingencia en el desempeño socioeconómico de los gobiernos locales}

El desafío de esta investigación es responder qué elementos caracterizan los factores de contingencia en el sector público y cómo influyen estos elementos en el desempeño socioeconómico municipal. Las principales hipótesis probadas en este estudio analizan si los factores de contingencia entorno (contexto externo), tecnología (procesos), estructura (inversiones) y porte (tamaño) influyen en el desempeño socioeconómico de los gobiernos locales. Para ello, se realizó una investigación documental con los 399 municipios del estado de Paraná, totalizando 1.995 observaciones para el período de 2013 a 2017, lo que corresponde a un ciclo presupuestario completo. Para el análisis de los datos se aplicó una regresión lineal múltiple con datos de panel. Los resultados revelan que los factores de contingencia entorno y porte inciden positivamente en los resultados socioeconómicos, mientras que el factor tecnología influye negativamente y el factor estructura no mostró resultados significativos. A partir de estos resultados se concluye que los factores externos, no controlables por los gestores, son los que mejor explican el comportamiento del desempeño socioeconómico de los gobiernos locales investigados. Así, corresponde a los gestores implementar políticas públicas capaces de interferir en su entorno y propiciar un porte que contribuya al desempeño socioeconómico y cumpla con los objetivos de la población. Asimismo, los resultados también tienen implicaciones teóricas, ya que aportan a la literatura en el sentido de sumar elementos que caracterizan las contingencias en el sector público, a nivel municipal, al flujo de investigación basada en la Teoría de la Contingencia, como el observatorio social, grado de dependencia, planificación presupuestaria, capacidades de la agencia pública para mantener sus gastos corrientes, estructura de personal e inversión y porte considerando los ingresos del municipio.

Palabras clave: factores de contingencia; desempeño socioeconómico; gobiernos locales. 


\section{Influence of contingency factors on the socioeconomic performance of local governments}

This research aims to identify which elements characterize the contingency factors in the public sector and how these elements influence municipal socioeconomic performance. The main hypotheses tested in this study analyze whether the contingency factors environment (external context), technology (processes), structure (investments), and size (population and revenues) influence the socioeconomic performance of local governments. We conducted documentary research with the 399 municipalities of Paraná, totaling 1,995 observations for the period from 2013 to 2017, which corresponds to a complete budget cycle. For data analysis, multiple linear regression with panel data was applied. The results reveal that the contingent factors environment and size positively affect the socioeconomic results, while technology has a negative influence and structure did not present significant results. Based on these results, we conclude that external factors, those not controllable by the managers, best explain the socioeconomic performance of the investigated local governments. Thus, managers need to implement public policies capable of impacting their environment and work towards achieving a population size able to improve socioeconomic performance and meets the population's objectives. As for theoretical implications, this study contributes to the literature by adding elements that characterize contingencies in the public sector, at the municipal level, to the flow of research based on contingency theory, such as social observatory, degree of dependence, budget planning, capacities of the public agency to maintain current expenses, personnel, and investment structure and size, when considering the municipality's revenue.

Keywords: contingency factors; socioeconomic performance; local governments.

\section{INTRODUÇÃO}

A Teoria da Contingência tem desafiado pesquisadores a encontrar elementos que materializem os fatores contingenciais no ambiente organizacional. Esse aspecto é observado num fluxo da pesquisa em contabilidade gerencial, que tem se dedicado a demonstrar o espectro do lastro de tal teoria em estudos dessa corrente. Otley (1980) salienta que, embora a área de estudos organizacionais venha discutindo fatores contingenciais desde o início da década de 1960, no ramo de sistemas, as abordagens se iniciaram apenas na década de 1970, sendo mais proeminente no fim dessa década a literatura que envolve aspectos organizacionais e comportamentais relacionados com os sistemas de controle gerencial. Na visão do autor, o cuidado em observar fenômenos organizacionais, como o uso de sistemas de controle gerencial atrelado ao desempenho numa perspectiva contingencial, contribuiu para o aprimoramento científico em investigações de cunho organizacional.

Nesse mesmo sentido, Chenhall (2003) demonstrou avanços significativos na discussão dos elementos que permeiam a Teoria da Contingência no campo organizacional, remetendo a um resgate dos fatores pesquisados por uma corrente de autores identificados numa revisão sistemática da literatura, como ambiente, tecnologia, estrutura, tamanho e estratégia.

Numa revisão sistemática da literatura de contabilidade gerencial lastreada em um ou mais fatores contingenciais, do período de 1980 a 2014, Otley (2016) levanta discussões contemporâneas sobre essa matéria, argumentando que a perspectiva contingencial mostra que quase sempre não há soluções universais para problemas no controle organizacional. Dessa maneira, a abordagem contingencial exige clareza conceitual para investigar aspectos organizacionais, em especial quando estes não apresentam congruências em suas manifestações ou não se revelam totalmente dominados pela literatura.

Avanços foram observados na abordagem contingencial em estudos organizacionais que discutem aspectos referentes aos sistemas de controle gerencial (Otley, 2016). As formulações de contingência se encontram subjacentes em estudos que contemplam tanto fatores internos quanto externos das 
organizações (Wadongo \& Abdel-Kader, 2014). Nota-se uma preocupação recorrente na literatura em relação aos efeitos de variáveis contingenciais no desempenho das organizações, uma vez que este é impactado por pressões internas e externas (Teisman \& Klijn, 2008). Observa-se, no entanto, uma prevalente preocupação com a caracterização dos fatores contingenciais em ambientes empresariais de natureza privada, que, conforme Woods (2009), apesar de apresentarem semelhanças ao setor público, se diferem no que concerne ao contexto, em comparação com o setor público.

Sell, Beuren e Lavarda (2020) apontam que a avaliação de desempenho se tornou especialmente destacada na nova gestão pública, com vistas no uso eficiente dos recursos públicos. Isso permite pesquisas para o entendimento de fatores contingenciais envoltos no setor público, com importantes efeitos no desempenho de governos. Sob essa visão, fazem-se as seguintes perguntas: quais elementos caracterizam os fatores contingenciais no setor público de governos locais? Eles influenciam o desempenho socioeconômico municipal? Assim, este artigo tem por objetivo verificar elementos representativos de fatores contingenciais no contexto do setor público e testar empiricamente sua influência no desempenho socioeconômico de governos locais.

Este estudo visa acrescentar à literatura sobre o espectro dos fatores contingenciais elementos que possam caracterizá-los no setor público, alinhados aos fatores já consumados na corrente teórica estudada no âmbito organizacional do setor privado. Outra contribuição se soma ao estudo de Sell et al. (2020), desenvolvido em municípios catarinenses, sobre a relação entre o desempenho de entes públicos e fatores contingenciais, pois, adicionalmente, o presente texto explora novos determinantes que caracterizam os fatores contingenciais no setor público, bem como o desempenho municipal.

Em comparação com o estudo de Sell et al. (2020), a presente pesquisa se diferencia quanto à formação do fator ambiente, no sentido de que, além do Índice de Desenvolvimento Humano Municipal (IDHM), acrescenta novos elementos, como o observatório social (instrumento de controle social externo aos municípios), o grau de dependência (quanto o município depende externamente de outros entes) e o Produto Interno Bruto (PIB). Portanto, somam-se essas características ao fator "ambiente externo" dos municípios. Outra diferença em relação ao estudo de Sell et al. (2020) consiste na inclusão do fator "tecnologia" na discussão de contingências no contexto organizacional público, já que conhecer os processos internos, sobretudo de planejamento, é oportuno para ampliar a discussão sobre fatores contingenciais. Destaca-se também a diferença em relação ao fator "estrutura", pois o estudo anterior considerou despesas com pessoal, enquanto neste se incluíram as despesas de capital (investimentos), na expectativa de que possam contribuir para explicar o referido fator contingencial.

Não obstante, outra contribuição se alinha à proxy "desempenho no setor público". No artigo anterior, foi utilizada a receita corrente líquida, enquanto neste se amplia tal discussão usando o indicador Firjan, que contempla uma série de questões fundamentais que se propõem a mensurar o desempenho municipal, como emprego, renda e investimentos em educação e saúde.

Dessa forma, busca-se contribuir para uma compreensão mais ampla dos efeitos que os elementos contextuais podem proporcionar à gestão de governos locais. A identificação de elementos representativos de fatores contingenciais no contexto do setor público e sua verificação empírica corroboram, junto às equipes estratégias dos órgãos públicos, no sentido de formular e implementar políticas com vistas a melhorar o desempenho socioeconômico. Nessa direção, pode-se coadunar com o viés prático-social da responsabilidade fiscal e da eficiência com o interesse público.

As seções que se seguem abordam o aporte teórico voltado à identificação dos elementos representativos de fatores contingenciais no contexto do setor público, associados ao desempenho 
socioeconômico no âmbito de governos locais. Suportam também as hipóteses da pesquisa e descrevem a operacionalização das variáveis no que concerne à sua verificação empírica. Por fim, evidenciam os resultados da pesquisa e apresentam implicações teóricas e práticas, visando colaborar para a compreensão do desempenho no setor público brasileiro, especificamente com governos locais, sob a lente de uma abordagem contingencial.

\section{APORTE TEÓRICO E HIPÓTESES DA PESQUISA}

A Teoria da Contingência, conforme Donaldson (2001), é uma das principais lentes teóricas para os estudos organizacionais, pois fornece várias ideias e suporte empírico. Segundo o autor, ela sustenta a relação entre a estrutura organizacional e contingências que impactam positivamente seu desempenho. No âmbito da contabilidade gerencial, pesquisas fundamentadas na Teoria da Contingência começaram a ser mais observadas por volta dos anos 1970, com vistas a compreender as variedades de práticas gerenciais emergentes, tendo como base fatores contingenciais, como aqueles relacionados com a estrutura organizacional (Otley, 2016).

Essa teoria se agrega a aspectos específicos de um sistema contábil, associados a determinadas circunstâncias, à sua apropriada correspondência, e caracterizados pelos chamados fatores contingenciais (Otley, 1980). Do estudo de Cadez e Guilding (2008), é possível extrair que os sistemas de controle gerencial e de desempenho organizacional, no contexto da formulação contingencial, são impactados por diferentes fatores específicos ou ambientais.

Em estudos de análise sistemática, como os realizados por Covaleski, Evans III, Luft e Shields (2003), bem como por Chenhall (2003) e Otley (2016), é possível observar algumas das principais variáveis externas e internas que permeiam as discussões pertinentes aos fatores contingenciais abordados na Teoria da Contingência, como as variáveis externas de ambiente e tecnologia e as variáveis internas relativas à estrutura e ao tamanho.

No que se refere ao ambiente organizacional, sob o enfoque da Teoria da Contingência, pode-se assumir que o progresso de determinada organização tende a se relacionar com as condições ambientais em que está inserida (Prajogo, 2016), especialmente ao observar as consideráveis incertezas (Chenhall, 2003; Otley, 2016). Dessa forma, as práticas organizacionais adotadas devem estar voltadas para melhorar o desempenho no ambiente em que está inserida, a fim de atingir o equilíbrio organizacional (Covaleski et al., 2003).

Nos entes públicos locais, alguns fatores ambientais tendem a afetar a realidade da gestão pública. Inicialmente, podem-se citar observatórios sociais (OS), os quais se caracterizam como um órgão externo de controle social, aproximam a sociedade do governo e contribuem para a qualidade da administração pública (Schommer \& Moraes, 2010). Outro fator que tende a influenciar a gestão dos governos locais é o Índice de Desenvolvimento Humano Municipal (IDHM), o qual trata de uma perspectiva social que contempla, entre outras coisas, questões ligadas a saúde, educação, emprego e renda (Mendes, Ferreira, Abrantes \& Farias, 2018), influenciando também o ambiente dos governos locais.

Além desses indicadores, o grau de dependência (GD) dos governos locais de transferências de recursos dos governos estaduais e federais pode denotar uma característica ambiental que impacta na gestão pública. Segundo Macedo e Corbari (2009), o grau de dependência tende a afetar o desempenho municipal. Por fim, com relação às variáveis ambientais que interferem no desempenho dos entes públicos, tem-se o PIB, que representa o crescimento econômico local (Mendes et al., 2018). 
Uma recente constatação empírica do estudo de Sell et al. (2020), que investigaram municípios catarinenses, indicou influência positiva do fator ambiente no desempenho municipal, o que se coaduna com o referencial teórico abordado. Assim, no que se refere ao fator contingencial ambiente, com base na discussão proposta antes, formula-se uma primeira hipótese: o fator contingencial ambiente influencia positivamente no desempenho socioeconômico dos governos locais.

Além dele, há o fator contingencial tecnologia, com significados diversos, entre os quais uma concepção geral que diz respeito a como os processos de trabalho da organização operam, como hardwares, softwares, materiais, pessoas e conhecimentos, o que pode responder às incertezas ambientais (Chenhall, 2003). De acordo com Otley (2016), o desenvolvimento das tecnologias impacta diretamente nas organizações, provocando mudanças, as quais geram incertezas ambientais.

No setor público, pode-se considerar um fator contingencial tecnologia o índice de realização das receitas orçamentária (Irro), que mede o nível de acerto do planejamento quanto à estimação das receitas orçamentárias, e o índice de execução orçamentária corrente (Ieoc), que mede o nível de capacidade de manter as despesas correntes (Santos \& Alves, 2011).

Tais indicadores se relacionam com os processos internos orçamentários praticados nas unidades públicas, ou seja, refletem como o fluxo de operações orçamentárias se comportam diante das atividades governamentais. Portanto, seguindo a lógica da premissa clássica contingencial (Chenhall, 2003), essas proxies se alinham ao fator tecnologia. Espera-se que a organização pública tenha boas práticas e eficiência em seus processos internos, impactando positivamente os indicadores socioeconômicos. Para tanto, formula-se uma segunda hipótese: o fator contingencial tecnologia influencia positivamente o desempenho socioeconômico dos governos locais.

Outro fator contingencial relevante é a estrutura da entidade. Segundo Donaldson (2001), há conexão de determinismo da estrutura pela contingência. Uma das proposições da Teoria da Contingência se refere à constatação de que o desempenho da organização está ligado ao ajuste entre o contexto e a estrutura organizacional (Cadez \& Guilding, 2008). Chenhall (2003) destaca que o fator contingencial estrutura pode afetar a eficiência e a motivação dos indivíduos, ajudando a moldar o futuro da organização.

Assim, para os entes públicos locais, observa-se como variáveis capazes de mensurar a estrutura o índice de despesas com pessoal (DP) e o índice de despesas com investimento (DI). O primeiro mede quanto da receita corrente líquida (RCL) municipal é comprometido para a manutenção de gastos com pessoal, enquanto o segundo tem como finalidade representar o nível de dispêndios com investimentos municipais em relação à sua RCL. Portanto, medir estrutura pelo viés de despesas correntes, como no caso dos gastos com servidores, e por despesas de capital, como os investimentos que representam obras e equipamentos, reflete a estrutura dos municípios (Macedo \& Corbari, 2009).

Sell et al. (2020), no mesmo sentido, relacionaram o fator estrutura, considerando despesas com pessoal, e encontraram influência positiva sobre o desempenho municipal. Diante das características das variáveis assumidas para representar o fator contingencial estrutura, formula-se a terceira hipótese: o fator contingencial estrutura influencia positivamente o desempenho socioeconômico dos governos locais.

O tamanho da organização afeta a estrutura burocrática (Donaldson, 2001) e tem certa imprevisibilidade associada a ele (Otley, 1980). Donaldson (2001) aduz que uma grande organização, com estrutura simples e inadequada, certamente encontrará uma gestão ineficaz, graças à sobrecarga das decisões a serem tomadas. Portanto, o tamanho da organização é capaz de impactar seu desempenho. 
Chenhall (2003) destaca que existem diferentes medidas capazes de representar o fator tamanho numa organização e que sua definição depende do contexto e das dimensões em estudo. No setor público, a receita municipal (REC) pode ser representativa do porte municipal. Segundo Vieira (2009), pode haver desigualdades no tamanho do setor público, se observadas pela óptica de receita. O porte populacional é outra variável que contempla características internas representativas do porte, capaz de impactar o desempenho (Cavalcante, 2016).

A densidade populacional como proxy de fator contingencial se apresentou com relação positiva sobre o desempenho municipal na pesquisa de Sell et al. (2020). Além disso, mensurar o porte pela receita municipal corrobora a discussão de evidenciações de contingências no setor público. Como visto no fluxo da literatura de contingência e no estudo ora citado, espera-se a relação descrita na quarta hipótese: o fator contingencial porte influencia positivamente o desempenho socioeconômico dos governos locais.

Diante das discussões teóricas apresentadas e das hipóteses formuladas, apresenta-se na sequência o delineamento metodológico da pesquisa.

\section{PROCEDIMENTOS METODOLÓGICOS}

\subsection{Delineamento da pesquisa e coleta de dados}

O delineamento metodológico assumido quanto aos objetivos é descritivo - pois se observam os fenômenos sem manipulá-los -, documental quanto aos procedimentos - com base em dados de fontes secundárias - e quantitativo em relação à abordagem do problema, em razão do uso de técnicas estatísticas para responder à pergunta da pesquisa. A amostra corresponde aos 399 municípios paranaenses. Presume-se que, como as características contingenciais assumidas (variáveis) são comuns em todos os municípios brasileiros, as interpretações dos índices se assemelhem, o que justifica a amostra para o teste empírico. Não obstante os demais municípios das outras unidades federativas, a escolha da presente pesquisa também ganhou força graças ao portal do Tribunal de Contas do Estado do Paraná (TCE-PR), que disponibiliza, de maneira consolidada e auditada, as informações de natureza orçamentária/financeira e contábil.

Os dados foram coletados no portal eletrônico do TCE-PR e do Instituto Brasileiro de Geografia e Estatística (IBGE), correspondentes ao ciclo eleitoral de 2013 a 2017. O período delimitado se justifica pelo fato de os dados financeiros e contábeis já se encontrarem consolidados e auditados pelos respectivos órgãos, o que denota maior confiabilidade nas 1995 observações deste estudo, além de representar um ciclo orçamentário completo para a análise do fenômeno alvo desta pesquisa.

\subsection{Variáveis e modelo econométrico de análise}

A operacionalização das variáveis é descrita no Quadro 1 com o intuito de atender às dimensões contingenciais tratadas nesta pesquisa. 


\section{QUADRO 1 VARIÁVEIS DA PESQUISA}

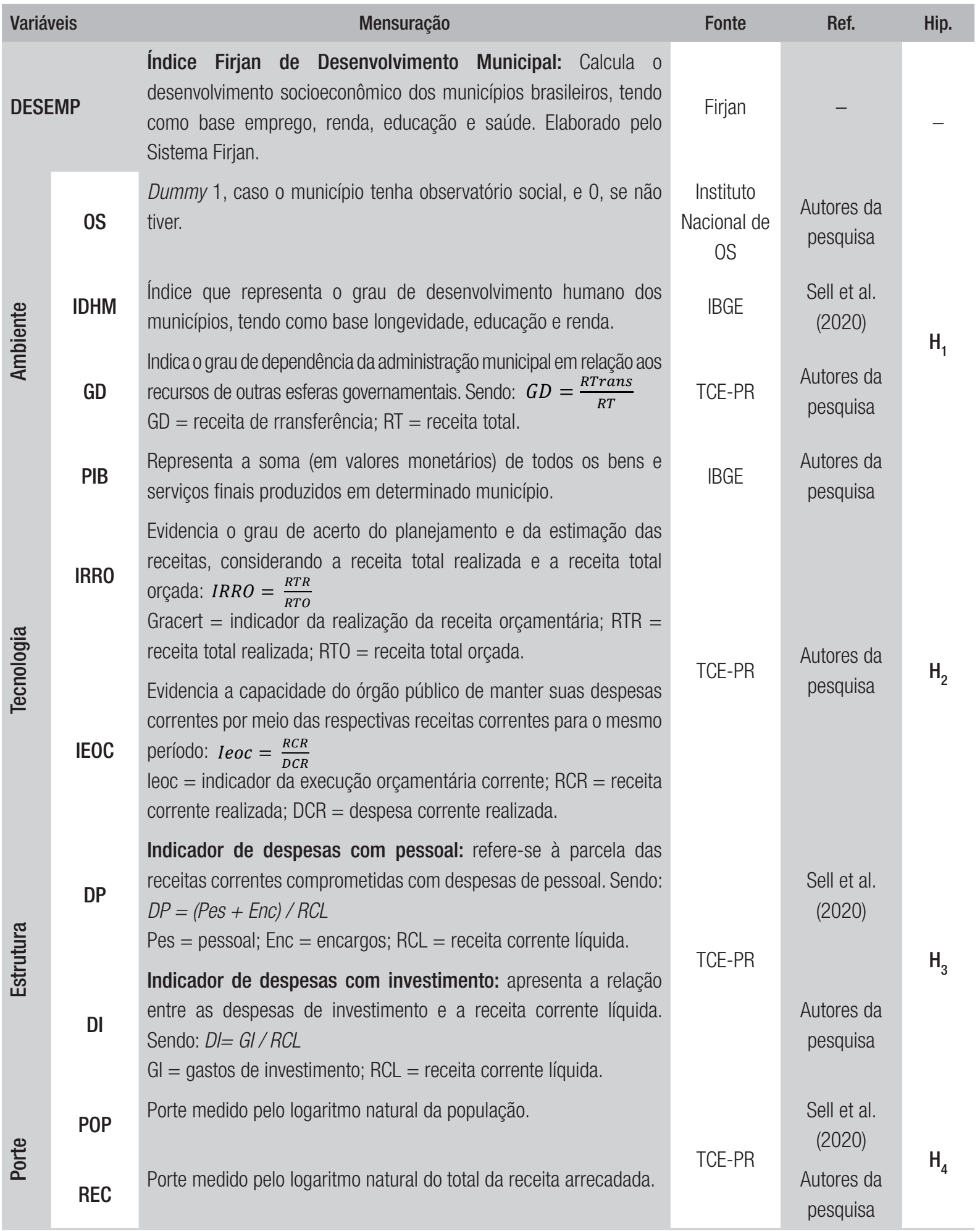

Fonte: Elaborado pelos autores. 
Em consonância com as hipóteses formuladas, na equação abaixo se apresenta o modelo econométrico para analisar a influência dos fatores contingenciais no desempenho socioeconômico dos municípios da amostra:

$$
\operatorname{DESEMP}_{I t}=\beta_{0 i t}+\beta_{1} A M B_{i t}+\beta_{2} T E C_{i t}+\beta_{3} E S T_{i t}+\beta_{4} P O R T E_{i t}+\epsilon_{i t}
$$

O agrupamento das variáveis em cada fator contingencial se valeu do método de avaliação do produto agregado ponderado (Waspas), que é uma combinação exclusiva do modelo de soma ponderada (WMG) e do modelo de produto ponderado (WPM). No Waspas, um critério conjunto de otimização é buscado com base em dois de otimalidade (Chakraborty, Zavadskas \& Antucheviciene, 2015). Para tanto, as variáveis de cada grupo pertencentes aos fatores contingenciais previstos no Quadro 1 - por exemplo, no fator ambiente se consideram as variáveis OS-IDHM-GD-PIB, e assim sucessivamente para cada contingência - foram consolidadas em seu respectivo fator, com base no método Waspas.

Os procedimentos de análise dos dados empregados neste estudo se valeram de técnicas estatísticas, como regressão linear múltipla com dados em painel, com o uso do software Stata versão 14. A modelagem em painel utilizada observou os testes de Breusch-Pagan, Chow e Hausman para delinear o modelo de regressão mais adequado para as observações em análise - efeitos fixos, aleatórios ou Pols (Greene, 2008).

\section{RESULTADOS DA PESQUISA}

Nesta seção, a descrição e a análise dos resultados foram organizadas de modo a exibir os aspectos relacionados com a robustez do modelo empírico aplicado e, na sequência, apresentar a discussão dos resultados dos testes das hipóteses da pesquisa.

\subsection{Robustez do Modelo Empírico e Descrição dos Resultados}

No sentido de proporcionar confiabilidade aos achados da pesquisa, na Tabela 1 se apresentam inicialmente os resultados do modelo proposto na equação acima e, na sequência, os pressupostos da regressão e a análise individual de cada fator contingencial.

TABELA 1 INFLUÊNCIA DOS FATORES CONTINGENCIAIS NO DESEMPENHO MUNICIPAL

\begin{tabular}{|c|c|c|c|c|c|c|c|}
\hline Prob $>F$ & $\mathrm{R}^{2}$ & DW & \multicolumn{2}{|l|}{ Mean VIF } & Teste BP/CW & Teste White & $\mathrm{N}^{\circ}$ obs \\
\hline 0.0000 & 0.0120 & 2.01 & \multicolumn{2}{|l|}{1.01} & 0.0000 & 1.400 & 1.995 \\
\hline \multirow{2}{*}{$\begin{array}{c}\text { Fatores } \\
\text { contingenciais }\end{array}$} & \multicolumn{7}{|c|}{ Efeitos fixos } \\
\hline & Coeficiente & Erro padrão & $\mathrm{T}$ & $P$-Value & VIF & \multicolumn{2}{|c|}{ Interv. Conf. 95\% } \\
\hline Ambiente & 0.0141 & 0.0057 & 2.48 & $0.013^{\star *}$ & 1.02 & 0.0030 & 0.0253 \\
\hline Tecnologia & -0.0002 & 6.6600 & -36.31 & $0.000^{\star \star \star}$ & 1.01 & -0.0003 & -0.0002 \\
\hline
\end{tabular}




\begin{tabular}{lcccccccc}
\multicolumn{1}{c}{$\begin{array}{c}\text { Fatores } \\
\text { contingenciais }\end{array}$} & Coeficiente & Erro padrão & T & P-Value & VIF & \multicolumn{2}{c}{ Interv. Conf. 95\% } \\
\hline Estrutura & -0.0104 & 0.0112 & -0.93 & 0.351 & 1.01 & -0.0323 & 0.0115 \\
Porte & 0.0112 & 0.0039 & 2.87 & $0.004^{\star \star \star}$ & 1.00 & 0.0035 & 0.0188 \\
Constante & 0.6834 & 0.0219 & 31.24 & 0.000 & - & 0.6405 & 0.7263 \\
\hline
\end{tabular}

Nota: Significância ao nível de até ${ }^{* *} 1 \% ;{ }^{* *} 5 \% ;{ }^{*} 10 \%$.

Legenda: Prob $>\mathrm{F}$ = significância do Modelo; $\mathrm{R}^{2}=$ poder explicativo do modelo; DW = Durbin-Watson - autocorrelação; Teste BP/CW = Breuch-Pagan/Cook-Weisberg - teste de heterocedasticidade dos resíduos; Teste White = teste de heterocedasticidade dos resíduos. Fonte: Dados da pesquisa.

A modelagem para verificar a influência dos fatores contingenciais sobre o desempenho socioeconômico se mostra consistente, pois os resultados dos testes de Breusch-Pagan, F de Chow e Hausman demonstram o uso de efeitos fixos para a amostra estudada, visto que o de Breusch-Pagan se apresentou menor que 0,05. Em consequência, aplicou-se o teste de Hausman, que foi menor que 0,05, condicionando a modelagem de painel de efeitos fixos. O modelo é significante ao nível de 1\%, com 1995 observações, o que corresponde a 399 municípios num lapso de cinco anos.

Não é propósito deste estudo encontrar variáveis explicativas para o desempenho socioeconômico dos municípios, e sim relacionar fatores contingenciais com o referido desempenho. Dessa maneira, as variáveis assumidas representam $1 \%\left(\mathrm{R}^{2}\right)$ de explicação em todo o contexto da performe mensurado pelo índice Firjan, pois essa métrica tem a finalidade de monitorar anualmente as principais áreas de desenvolvimento municipal, em especial emprego e renda, educação e saúde (Avelino, Gama \& Cunha 2013).

Os resultados expressam que o modelo não viola os pressupostos de autocorrelação e multicolinearidade, pois a estatística Durbin-Watson $(2,01)$ está próxima de 2 e os valores do VIF $(1,16<5)$ se apresentam menores que 5 . Observa-se, no teste de White, que o modelo apresenta problemas de heterocedasticidade. No entanto, para proporcionar confiabilidade aplicou-se a correção robusta de White.

Numa forma panorâmica, o modelo não viola os pressupostos básicos exigidos na regressão múltipla, o que permite avançar na análise de cada fator contingencial (variável), coadunada com a discussão das hipóteses. Feita a análise da robustez do modelo proposto, na Tabela 1 se apresentam os resultados da influência de cada variável do respectivo fator contingencial sobre o desempenho socioeconômico dos governos locais pesquisados.

\subsection{Discussão dos Resultados}

Para a discussão dos resultados da pesquisa, no Quadro 2 se demonstram as decisões para as hipóteses da pesquisa. 


\section{QUADRO 2 DECISÃO PARA AS HIPÓTESES TESTADAS}

\begin{tabular}{cll} 
Hipóteses & \multicolumn{1}{c}{ Relações propostas } & \multicolumn{1}{c}{ Decisões } \\
$\mathrm{H}_{1}$ & Fator contingencial ambiente $\rightarrow$ Desempenho socioeconômico & Confirmada \\
$\mathrm{H}_{2}$ & Fator contingencial tecnologia $\rightarrow$ Desempenho socioeconômico & Não confirmada \\
$\mathrm{H}_{3}$ & Fator contingencial estrutura $\rightarrow$ Desempenho socioeconômico & Não significante (NS) \\
$\mathrm{H}_{4}$ & Fator contingencial porte $\rightarrow$ Desempenho socioeconômico & Confirmada
\end{tabular}

Fonte: Dados da pesquisa.

O primeiro fator contingencial analisado, que representa o ambiente, tendo como variáveis representativas OS, IDHM, GD e PIB, influencia positivamente o desempenho socioeconômico dos governos locais investigados. Os dados agrupados por meio dos métodos utilizados das variáveis descritas demonstram que o índice de desempenho tende a aumentar na medida em que tais variáveis se apresentam positivas, o que denota efeitos de fatores externos na gestão dos municípios analisados. Assim, os achados da pesquisa permitem confirmar a hipótese $\mathrm{H}_{1}$ e contribuem para a compreensão de que os fatores ambientais são determinantes para a análise do desempenho socioeconômico municipal.

Esses fatores foram objeto de estudos empíricos anteriores, como o de Schommer e Moraes (2010), que afirmam que observatórios sociais podem ser determinantes para a administração dos entes públicos. Mendes et al. (2018) constataram que o IDH é uma perspectiva social relacionada com indicadores de saúde, educação e renda, assim como o PIB representa o crescimento econômico, ambos capazes de impactar a gestão do ente.

Macedo e Corbari (2009) verificaram que a variável do grau de dependência, oriunda de transferências intergovernamentais, é um fator que impacta o desempenho dos governos locais. Estudos seminais da área de contabilidade gerencial que abordaram a Teoria da Contingência, como de Otley (1980), Chenhall (2003) e Covaleski et al. (2003), já alertavam que o fator ambiental tende a impactar o desempenho das organizações. Os achados do presente estudo relativos ao fator ambiente convergem com o de Sell et al. (2020), ampliando os limites ao considerar outras características que reforçam a relação entre ambiente e desempenho municipal.

No segundo fator contingencial analisado, tecnologia, representado pelas variáveis índice de realização da receita corrente e índice de execução orçamentária corrente, os resultados revelam que os parâmetros associados às variáveis tecnologia apresentam influência negativa sobre o desempenho socioeconômico municipal. Portanto, os achados da pesquisa para o conjunto de dados observados e variáveis admitidas na pesquisa sugerem um resultado diferente do esperado, ou seja, a influência do fator contingencial representativo da tecnologia é negativa em relação ao desempenho municipal, o que leva à não confirmação da hipótese $\mathrm{H}_{2}$.

Embora bastante significativo, ressalta-se que o coeficiente associado aos resultados da regressão é baixo, o que pode estar ligado às características das variáveis admitidas para a representação desse fator contingencial. Apesar de as variáveis estarem associadas à capacidade de planejamento e manutenção das atividades, conforme destacado por Santos e Alves (2011), a busca pelo equilíbrio das contas públicas em conformidade com as diretrizes da Lei de Reponsabilidade Fiscal, no que se 
refere ao planejamento e à execução orçamentária, pode justificar o resultado obtido. A influência negativa das variáveis que compõem o fator tecnologia sobre o desempenho municipal demonstra que os processos internos de gestão pública necessitam de mais constatações empíricas, pois no setor público ainda prevalece o atendimento de limites legais como propósito principal de gestão, e não de fato um nível de serviço qualificado como contraprestação ao interesse público.

No que se refere ao terceiro fator contingencial observado, associado às variáveis das despesas municipais com pessoal e investimentos, os resultados não foram significativos. Portanto, não há suporte para inferir informações a respeito da hipótese $\mathrm{H}_{3}$, que previa influência positiva desse fator sobre o desempenho socioeconômico dos municípios paranaenses. Conjetura-se que, mesmo o p-value não apresentando significância, as características de estrutura apresentam influência sobre o desempenho, uma vez que a variável despesas com pessoal já foi testada empiricamente no estudo de Sell et al. (2020) como elemento do fator estrutura. Porém, graças à limitação da amostra assumida, as observações não foram suficientes para gerar um resultado com significância estatística.

Por fim, o quarto fator contingencial observado está relacionado com o tamanho dos municípios, as variáveis de porte (representativa da população) e a receita municipal. Pelos resultados, o fator contingencial porte confirma a hipótese $\mathrm{H}_{4}$, tendo em vista que os achados indicam que, quanto maior for o ente, maior será seu desempenho socioeconômico. Decorre que municípios com maior alavancagem, especialmente no que se refere às receitas, atrelada a um maior índice populacional, têm melhores condições de investir nas áreas da saúde, educação e renda, observadas pelo indicador de desempenho socioeconômico admitido nesta pesquisa. Esse achado ratifica Sell et al. (2020), que também encontraram relação entre fator porte e desempenho municipal.

Estudos empíricos, como os de Vieira (2009), para o caso das receitas, e de Cavalcante (2016), para o caso do tamanho da população, assumem que o porte dos municípios varia significativamente, o que acaba impactando no desempenho dos governos locais. Em consonância com o preconizado na Teoria da Contingência, Donaldson (2001) e Chennal (2003) destacam a importância de observar essas variáveis e de utilizar parâmetros adequados para a sua mensuração.

Depreende-se, porém, que os fatores contingenciais ambiente, tecnologia e estrutura sugerem explicar o desempenho socioeconômico dos municípios pesquisados, sendo que o ambiente e a estrutura influenciam positivamente, e a tecnologia, negativamente. Dessa maneira, confirmam-se as proposições contidas nas hipóteses $\mathrm{H}_{1}$ e $\mathrm{H}_{4}$ e não se confirma a contida na hipótese $\mathrm{H}_{2}$. No que se refere à estrutura municipal, os resultados não permitem fazer inferências estatísticas significativas.

Com os achados desta pesquisa, podem-se fazer análises relevantes para a prática gerencial dos municípios, já que os resultados sugerem melhor desempenho socioeconômico quando os municípios têm ambiente e porte favoráveis. Assim, a adoção de mecanismos adequados - voltados ao controle e à fiscalização externa -, políticas públicas em áreas de interesse social, otimização das receitas e políticas voltadas à manutenção da população local podem contribuir para melhores resultados em termos de desempenho socioeconômico. 


\section{CONSIDERAÇÕES FINAIS}

Este estudo investigou elementos representativos de fatores contingenciais no contexto do setor público e testou empiricamente sua influência no desempenho socioeconômico de governos locais. Os resultados sugerem que os fatores contingenciais associados ao ambiente e ao porte dos municípios influenciam positivamente o desempenho socioeconômico dos governos locais, alinhado com o proposto nas hipóteses $\mathrm{H}_{1}$ e $\mathrm{H}_{4}$. No que se refere ao fator tecnologia, os achados não confirmaram a hipótese $\mathrm{H}_{2}$, haja vista que indicou influência negativa desse fator no desempenho. Já o fator contingencial estrutura não apresentou significância estatística, portanto não suportou a confirmação da hipótese $\mathrm{H}_{3}$. Conclui-se, com base nesses resultados, que os fatores externos, aqueles que não podem ser diretamente controlados pelos gestores, são os que melhor explicam o comportamento do desempenho socioeconômico dos governos locais investigados.

Implicações teóricas advêm dos resultados da pesquisa ao analisar os efeitos dos fatores contingenciais no desempenho num campo pouco explorado na literatura gerencial. Dessa maneira, o estudo contribui para a literatura no sentido de acrescentar ao fluxo da pesquisa fundamentada na Teoria da Contingência elementos que caracterizam contingências no setor público, no âmbito municipal, como observatório social, grau de dependência, planejamento orçamentário, capacidades do órgão público em manter suas despesas correntes, estrutura de pessoal e de investimentos e porte considerando a receita do município. Em especial, o observatório social se mostra um dos elementos interessantes para a verificação de contingências, pois esse controle social nos municípios pode influenciar o desempenho das condições socioeconômicas.

Os resultados do estudo também apresentam contribuições prático-sociais, em especial para a gestão pública dos entes quanto aos indicadores de desempenho socioeconômico locais, associados a variáveis representativas dos fatores contingenciais. Ao considerar os fatores ambiente, tecnologia, estrutura e porte, conseguiu-se extrair os grupos capazes de explicar e determinar o desempenho. Os resultados contribuem especialmente ao revelar que fatores externos, como o ambiente em que os governos estão inseridos, e aqueles relacionados com o seu porte impactam positivamente os índices de desenvolvimento municipal, bem como que os fatores representativos dos processos internos, como a tecnologia, com resultado negativo, e a estrutura que não foi significativa não se coadunaram com a influência previamente esperada no contexto do setor público. Portanto, cabe aos gestores implantar políticas públicas capazes de interferir em seu ambiente e incentivar um porte que contribua para o desempenho socioeconômico e atenda aos objetivos da população.

Os resultados desta pesquisa, no entanto, se limitam às variáveis assumidas para determinar os fatores contingenciais. Assim, recomenda-se que futuros estudos considerem outras variáveis para delinear os fatores contingenciais, de modo a ampliar o escopo das investigações sobre tal temática no setor público. Outra limitação da pesquisa decorre dos métodos usados para a agregação das variáveis no respectivo grupo, o que permite futuros estudos que sejam capazes de associar as variáveis aos respectivos fatores contingenciais. Ademais, diferentes medidas de desempenho podem ser selecionadas como indicativas do desempenho de governos locais, como a capacidade de geração de receita própria do município e índice de satisfação da população com os serviços públicos planejados e ofertados durante o ciclo orçamentário do plano plurianual. Recomenda-se ainda que se investigue a influência desses fatores no bem-estar social da população. 


\section{REFERÊNCIAS}

Avelino, B. C., Bressan, V. G. F., \& Cunha, J. V. A. (2013). Estudo sobre os fatores contábeis que influenciam o Índice Firjan de Desenvolvimento Municipal (IFDM) nas capitais brasileiras. Revista de Educação e Pesquisa em Contabilidade, 7(3), 274-290.

Burns, T., \& Stalker, G. (1961). The management of innovation. London, UK: Tavistock.

Cadez, S., \& Guilding, C. (2008). An exploratory investigation of an integrated contingency model of strategic management accounting. Accounting, Organizations and Society, 33(7-8), 836-863.

Cavalcante, P. (2016). Desempenho fiscal e eleições no Brasil: uma análise comparada dos governos municipais. Revista de Administração Pública, 50(2), 307-330.

Chakraborty, S., Zavadskas, E. K., \& Antucheviciene, J. (2015). Applications of waspas method as a multi-criteria decision-making tool. Economic Computation \& Economic Cybernetics Studies \& Research, 49(1), 5-22.

Chenhall, R. H. (2003). Management control systems design within its organizational context: findings from contingency-based research and directions for the future. Accounting, Organizations and Society, 28(2-3), 127-168.

Covaleski, M. A, Evans III, J. H, Luft, J. L, \& Shields, M. D (2003). Budgeting research: three theoretical perspectives and criteria for selective integration. Journal of Management Accounting Research, 15 (1), 3-49.

Donaldson, L. (2000). Organizational portfolio theory: performance-driven organizational change. Contemporary Economic Policy, 18(4), 386-396.

Donaldson, L. (2001). The contingency theory of organizations. Thousand Oaks, CA: Sage.

Galbraith, J. (1973). Designing complex organizations. Boston, MA: Addison Wesley Publishing Company.

Greene, W. H. (2008). The econometric approach to efficiency analysis. In H. O. Fried, C. A. K. Lovell, \& S. S. Schmidit (Org.), The measurement of productive efficiency and productivity growth. Oxford, UK: Oxford University Press.

Lawrence, P., \& Lorsch, J. (1967). Organization and environment. Homewood, IL: Irwin.
Macedo, J., \& Corbari, E. C. (2009). Efeitos da Lei de Responsabilidade Fiscal no endividamento dos municípios brasileiros: uma análise de dados em painéis. Revista Contabilidade \& Finanças, 20(51), 44-60.

Mendes, W. A., Ferreira, M. A. M., Abrantes, L. A., \& Faria, E. R. (2018). A influência da capacidade econômica e da formação de receitas públicas no desenvolvimento humano. Revista de Administração Pública, 52(5), 918-934.

Otley, D. (1980). The contingency theory of management accounting: achievement and prognosis. Accounting, Organizations and Society, 5(4), 413-428.

Otley, D. (2016). The contingency theory of management accounting and control (1980-2014). Management Accounting Research, 31(2016), 45-62.

Perrow, C. (1970). Organizational analysis: a sociological view. Belmont, CA: Wadsworth Publishing Company.

Prajogo, D. I. (2016). The strategic fit between innovation strategies and business environment in delivering business performance. International Journal of Production Economics, 171(2016), 241249.

Santos, S. R. T. D., \& Alves, T. W. (2011). O impacto da Lei de Responsabilidade Fiscal no desempenho financeiro e na execução orçamentária dos municípios no Rio Grande do Sul de 1997 a 2004. Revista de Administração Pública, 45(1), 181-208.

Schommer, P. C., \& Moraes, R. L. (2010). Observatórios sociais como promotores de controle social e accountability: reflexões a partir da experiência do observatório social de Itajaí. Gestão. org, 8(3), 298-326.

Sell, F. F., Beuren, I. M., \& Lavarda, C. E. F. (2020). Influência de fatores contingenciais no desempenho municipal: evidências inferenciais. RCO - Revista de Contabilidade e Organizações, 14, e164058.

Teisman, G. R., \& Klijn, E. (2008). Complexity theory and public management. Public Management Review, 10(3), 287-297.

Thompson, J. D. (1967). Organizations in action. New York, NY: McGraw Hill.

Vieira, R. D. S. (2009). O tamanho do setor público no contexto do federalismo: um modelo aplicado 
aos municípios brasileiros (Texto para Discussão n ${ }^{\circ}$ 1415). Brasília, DF: Ipea.

Wadongo, B., \& Abdel-Kader, M. (2014). Contingency theory, performance management and organizational effectiveness in the third sector: a theoretical framework. International Journal of
Productivity and Performance Management, 63(6), 680-703.

Woods, M. (2009). A contingency theory perspective on the risk management control system within Birmingham City Council. Management Accounting Research, 20(1), 69-81.

\section{Clóvis Fiirst}

https://orcid.org/0000-0002-9415-104X

Mestre em Ciências Contábeis e Administração pela Universidade Regional de Blumenau (FURB); Professor do Departamento de Ciências Contábeis da Universidade Estadual do Oeste (UNIOESTE).

E-mail: cfiirst@gmail.com

\section{Ilse Maria Beuren}

https://orcid.org/0000-0003-4007-6408

Doutora em Controladoria e Contabilidade pela Universidade de São Paulo (FEA/USP); Professora Permanente do Programa de Pós-graduação em Contabilidade da Universidade Federal de Santa Catarina (PPGC/UFSC). E-mail: ilse.beuren@gmail.com 